\title{
A simple non-equilibrium bedload transport equation for the formation of dune in a shallow- water flow over an erodible bed
}

\author{
Pablo Cañada-Pereira ${ }^{1, *}$ and Patricio Bohorquez ${ }^{2, * *}$ \\ ${ }^{1}$ Departamento de Ingeniería Mecánica y Minera, University of Jaén, 23071 Jaén, Spain \\ ${ }^{2}$ Centro de Estudios Avanzados en Ciencias de la Tierra, University of Jaén, 23071 Jaén, Spain
}

\begin{abstract}
In this work, we consider the long-standing problem of capturing dune formation in an erodible-bed channel at subcritical speed by using a reduced order model of depth-averaged equations. The pioneering study by Reynolds [1] showed that the standard Saint-Venant-Exner equations are unconditionally stable at subcritical Froude number. Hence, the use of depthaveraged flow equations, which are commonly used by the hydraulic community, prevents the formation of bedforms as dunes. Recently, Cañada-Pereira \& Bohorquez [2] have proposed a simple sediment transport formulation able to capture the formation of dune when coupled with the Saint-Venant equations. We replace the standard Exner equation with a non-equilibrium sediment transport equation that includes the following necessary ingredients: first, a phase shift in the particle entrainment rate; second, a particle diffusivity and an eddy viscosity. Subsequently, we solve the linear stability problem of an erodiblebed channel and show that the neutral curve properly captures the bed instability both in subcritical regime (i.e. dune) and supercritical flow (i.e. antidune and roll wave). Finally, we corroborate the capabilities of the model by means of non-linear numerical simulations which reproduce the growth of dune and antidune in agreement with experiments.
\end{abstract}

\section{Introduction}

The prediction of the occurrence of bedforms and its morphology has been a case of study of great interest due to its influence in major engineering problems such as floods and the effects on the landscape in general. The main theoretical frameworks used to study this problem can be classified, according to the flow equations, into rotational formulations using NavierStokes equations, and its depth-integrated form known as the shallow water equations (also referred to as Saint-Venant equations). Morphodynamic models add supplementary equations that describe sediment transport processes [3].

The Navier-Stokes approach has proven to be a good option to predict with accuracy the evolution of these phenomena, whose standardised numerical scheme and closure equations provide reliable results checked with experimental data. Even though a rotational formulation

\footnotetext{
*e-mail: pcanada@ujaen.es

**e-mail: patricio.bohorquez@ujaen.es
} 


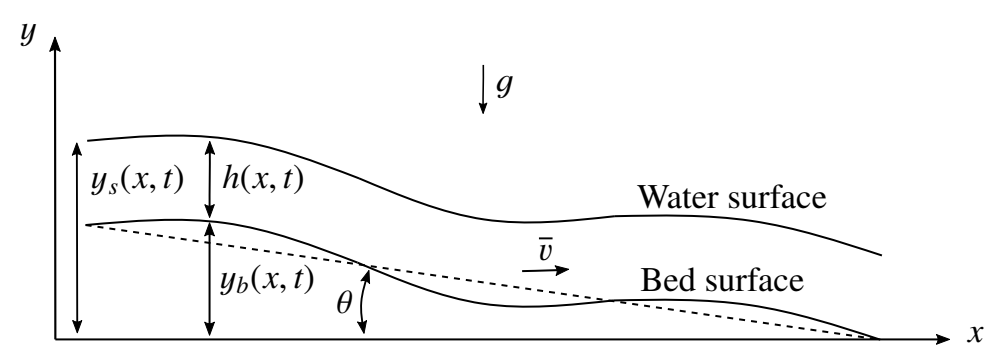

Figure 1. Sketch of an open channel flow over an erodible bed.

seems to be a great solution, one of its major drawbacks lies in the huge computational cost that confines its use rather in supercomputers or for a simplified and low-resolution computational domain. Within this strategy, many studies have been carried out, such as Niemann et al. [4] and Van Duin et al. [5]. Alternative morphodynamic models for dune formation based on the Saint-Venant equations are scarce and require an ad-hoc bed slope correction term that triggers the instability of the bed $[6,7]$. Hence, existing morphodynamic models predict the formation of bedforms with precision at the expense of relatively high computational cost or approximately using some ad-hoc formulation of physical processes to trigger the instabilities.

An alternative approach was first introduced by Kennedy [8] and continued by other authors such as Charru et al. [9] who proposed the incorporation of a phase shift to the transport rate and the shear stress, respectively. This study is motivated by the need to set a simple and standard model that accurately and efficiently describes the instability of an erodible bed at the inception of sediment motion. Hence, we extend the one-dimensional morphodynamical model of shallow-water flow over an inclined erodible bed by Bohorquez \& Ancey [10, 11], which couples the Saint-Venant equation of hydraulic engineering to a bed-load model advocated by Ancey \& Heyman [12], to predict the dune/antidune instability of the erodible bed as well as the roll wave instability of the free surface. The key point is the inclusion of a phase lag between the sediment entrainment rate and the depth-averaged flow velocity, as originally proposed by Cañada-Pereira \& Bohorquez [2].

\section{Formulation of the problem}

Figure 1 shows a simplified representation of the problem we address and the main variables that affect the morphology of bedforms and water waves. The depth-averaged velocity is denoted by $\bar{v}\left[\mathrm{~m} \mathrm{~s}^{-1}\right]$ and the flow depth is given by $h(x, t)=y_{s}-y_{b}$ with $y_{b}(x, t)[\mathrm{m}]$ and $y_{s}(x, t)[\mathrm{m}]$ being the bed and free surface elevations, respectively. As usual, $t[\mathrm{~s}]$ is time, $x[\mathrm{~m}]$ is the streamwise coordinate, $g\left[\mathrm{~m} \mathrm{~s}^{-2}\right]$ represents the gravitational acceleration, and the bed slope is defined as $\tan \theta=-\partial_{x} y_{b}$. The governing equations of the water depth, speed and the height of the bed can be written in the dimensional form as [11, 12]:

$$
\begin{gathered}
\frac{\partial h}{\partial t}+\frac{\partial h \bar{v}}{\partial x}=0, \\
\frac{\partial h \bar{v}}{\partial t}+\frac{\partial h \bar{v}^{2}}{\partial x}+g h \frac{\partial h}{\partial x}=-g h \frac{\partial y_{b}}{\partial x}-\frac{\tau_{b}}{\rho}+\frac{\partial}{\partial x}\left(v h \frac{\partial \bar{v}}{\partial x}\right), \\
\left(1-\zeta_{b}\right) \frac{\partial y_{b}}{\partial t}=\kappa \gamma-\lambda .
\end{gathered}
$$


The proposed equations correspond to the standard mass (1) and momentum (2) conservation equations by Saint-Venant, and a modified Exner equation (3), where the term of the divergence of sediment flux has been substituted by a non-equilibrium entrainment-deposition rate expression $\left(-\partial_{x} q_{s}=\kappa \gamma-\lambda\right)$. The head loss induced by hydraulic resistance, $\tau_{b}$, is evaluated as $\tau_{b} / \rho=f / 8 \bar{v}|\bar{v}|$ where $f$ is the dimensionless Darcy-Weisbach friction factor. The extra term, $\partial_{x}\left(v h \partial_{x} \bar{v}\right)$, represents a depth-averaged Reynolds stress where $v$ is the eddy viscosity $\left[\mathrm{m}^{2} \mathrm{~s}^{-1}\right]$. In (3), $\zeta_{b}$ is the dimensionless bed porosity, $\kappa\left[\mathrm{s}^{-1}\right]$ and $\lambda\left[\mathrm{m} \mathrm{s}^{-1}\right]$ represent the deposition and entrainment rates, respectively.

The Saint-Venant-Exner equations (1)-(3) are supplemented by an advection-diffusion equation for the particle activity, $\gamma(x, t)$ [m] (solid volume of particles in motion per unit streambed area), that reads $[10,13]$

$$
\frac{\partial \gamma}{\partial t}+\frac{\partial \bar{u}_{s} \gamma}{\partial x}-\frac{\partial^{2} D_{u} \gamma}{\partial x^{2}}=\lambda-\kappa \gamma
$$

where $D_{u}\left[\mathrm{~m}^{2} \mathrm{~s}^{-1}\right]$ denotes the particle diffusivity, and $\bar{u}_{s}=\beta \bar{v}\left[\mathrm{~m} \mathrm{~s}^{-1}\right]$ is the mean velocity of moving particles, with the constant coefficient in the range of $\beta \leq 1$.

The closure equation for the Darcy-Weisbach friction factor, $f$, is evaluated from Colebrook equation considering fully developed turbulent flow and rough regime,

$$
\frac{1}{\sqrt{f}}=-2 \log _{10}\left(\frac{\hat{d}}{3.71}\right)
$$

in which $\hat{d}=d / h$ is the grain roughness relative to flow depth. An approximate estimation of eddy viscosity is given by the mixing length equation $v=v_{t} h\left(f \bar{v}^{2} / 8\right)^{1 / 2}$ with the dimensionless parameter $v_{t} \sim O(1)$. The precise dependence of particle diffusivity $D_{u}$ on the flow variables remains unknown but can be related to the eddy viscosity using the turbulent Schmidt number, $\mathrm{Sc}=v / D_{u}$, which has been set up to $\mathrm{Sc}=0.5$. For the sake of simplicity, we set $v_{t}=1$ and $\beta=1$. Furthermore, $\kappa$ is assumed constant, and $\lambda$ is evaluated as established in Bohorquez \& Ancey [10], Lajeunesse et al. [14] and also Charru et al. [9].

\section{Linear stability theory}

With a suitable scaling with respect to a length scale $h_{0}$, a velocity scale $\bar{v}_{0}$, and a characteristic particle activity $\gamma_{0}$, corresponding to a uniform flow, the dimensionless governing equations have been obtained by making the following change of variables:

$$
u \leftarrow \frac{\bar{v}}{\bar{v}_{0}}, \quad \eta \leftarrow \frac{h}{h_{0}}, \quad z \leftarrow \frac{y_{b}}{h_{0}}, \quad \phi \leftarrow \frac{\gamma}{\gamma_{0}}, \quad \hat{x} \leftarrow \frac{x}{h_{0}}, \quad \hat{t} \leftarrow \frac{t \bar{v}_{0}}{h_{0}} .
$$

Note that the subscript 0 denotes the value of the base flow from now on. Furthermore, to simplify the notation, we introduce the equivalent bed slope of the base flow as $S_{0}=f_{0} v_{0}^{2} /\left(8 g h_{0}\right)$ and recall that $\gamma_{0}=\lambda_{0} / \kappa$. These base flow conditions are not limited necessarily to a sloped terrain but can be easily used in other phenomena as dam-break and glacial outburst floods under quasi-uniform flow and quasi-steady regime.

The linear perturbation equations are obtained by the standard procedure of asymptotic expansion. Hence, we decompose the perturbed variable as the sum of the background flow plus a small amplitude disturbance, i.e. $(z, \phi, \eta, u)=\left(-\hat{x} S_{0}, 1,1,1\right)+\epsilon\left(z^{\prime}, \phi^{\prime}, \eta^{\prime}, u^{\prime}\right)$. Substituting the decomposition into (1)-(4), expanding in Taylor series the friction factor as a function of the dimensionless grain roughness $\hat{d}_{0}$ (see (5)), and the dimensionless saturated (or equilibrium) particle activity $\phi_{e q}=\lambda / \lambda_{0}$ about the base flow, retaining only the terms of 
the order $O(\epsilon)$ and removing the prime decoration, we end up with the linear perturbation equations:

$$
\begin{gathered}
\frac{\partial \eta}{\partial \hat{t}}+\frac{\partial \eta}{\partial \hat{x}}+\frac{\partial u}{\partial \hat{x}}=0 \\
\operatorname{Fr}^{2}\left(\frac{\partial u}{\partial \hat{t}}+\frac{\partial u}{\partial \hat{x}}\right)+\frac{\partial \eta}{\partial \hat{x}}-\operatorname{Fr}^{2} \hat{v} \frac{\partial^{2} u}{\partial \hat{x}^{2}}=-\frac{\partial z}{\partial \hat{x}}+\eta S_{0}-2 u S_{0}, \\
\frac{\partial z}{\partial \hat{t}}=\hat{\kappa}_{e}\left(\phi-u^{\varphi} \frac{\mathrm{d} \phi_{e q}}{\mathrm{~d} u}\right) \\
\frac{\partial \phi}{\partial \hat{t}}+\beta\left(\frac{\partial u}{\partial \hat{x}}+\frac{\partial \phi}{\partial \hat{x}}\right)-\hat{D}_{u} \frac{\partial^{2} \phi}{\partial \hat{x}^{2}}=\hat{\kappa}\left(u^{\varphi} \frac{\mathrm{d} \phi_{e q}}{\mathrm{~d} u}-\phi\right) .
\end{gathered}
$$

The derivative term resulting from the Taylor series expansion of $f$ was neglected due to $\hat{d}_{0} \ll 1$.

The dimensionless parameters in (7)-(10), denoted below by a circumflex, are related with the dimensional quantities by means of the following scales:

$$
\mathrm{Fr}=\frac{\bar{v}_{0}}{\sqrt{g h_{0}}}, \quad \hat{D}_{u}=\frac{D_{u}}{h_{0} \bar{v}_{0}}, \quad \hat{v}=\frac{v}{h_{0} \bar{v}_{0}}, \quad \hat{\kappa}_{e}=\frac{\kappa \gamma_{0}}{\left(1-\zeta_{b}\right) \bar{v}_{0}}, \quad \hat{\kappa}=\frac{\kappa h_{0}}{\bar{v}_{0}} .
$$

According to Section 2, the dimensionless deposition rate reads $\hat{\kappa}=c_{d}(s-1)^{1 / 2} /\left(\operatorname{Fr} \hat{d}_{0}^{1 / 2}\right)$, with $c_{d}=0.1$ (coefficient inferred from the particle flight time). The relative density mismatch for silt in water is typically $s=2.65$. Also, the proposed entrainment rate function is given by $\hat{\kappa}_{e}=14 \hat{\kappa} \hat{d}_{0}\left(\mathrm{Sh}-\mathrm{Sh}_{c r}\right)$. Here, Sh denotes the Shields number which represents the dimensionless shear stress:

$$
\mathrm{Sh}=\frac{f \mathrm{Fr}^{2}}{8(s-1) \hat{d}_{0}} u^{2} .
$$

The threshold value of motion for sand and gravel in hydraulically rough turbulent flow is typically $\mathrm{Sh}_{c r}=0.02$ (from Julien [15]). A suitable empirical relation for particle activity in uniform flow arises from the relations used for the entrainment and deposition rates,

$$
\phi_{e q}(\mathrm{Sh})= \begin{cases}\left(\mathrm{Sh}-\mathrm{Sh}_{c r}\right) /\left(\mathrm{Sh}_{0}-\mathrm{Sh}_{c r}\right) & \text { if } \mathrm{Sh} \geq \mathrm{Sh}_{c r} \\ 0 & \text { if } \mathrm{Sh}<\mathrm{Sh}_{c r}\end{cases}
$$

being $\mathrm{Sh}_{0}$ the value of the Shields number corresponding to the base flow. Therefore, the derivative term resulting from Taylor expansion of $\phi_{e q}$ in (9) and (10) yields:

$$
\phi_{e q}^{\prime}=\left.\frac{\mathrm{d} \phi_{e q}}{\mathrm{~d} u}\right|_{\substack{u=1 \\ \eta=1}}=\frac{\mathrm{d} \phi_{e q}}{\mathrm{dSh}} \frac{\mathrm{dSh}}{\mathrm{d} u}=\frac{2 \mathrm{Sh}_{0}}{\mathrm{Sh}_{0}-\mathrm{Sh}_{c r}}
$$

Finally, the solution to (7)-(10) can be written as $(z, \phi, \eta, u)=\mathbf{T} \exp [i(\hat{k} \hat{x}-\hat{\omega} \hat{t})]$, where the eigenvector is denoted by $\mathbf{T} \equiv(\zeta, \Phi, \Gamma, U)^{T}$, the dimensionless wavenumber by $\hat{k}$, and the dimensionless complex frequency by $\hat{\omega}=\hat{\omega}_{r}+i \hat{\omega}_{i}$ (Schmidt \& Henningson [16]).

A key part of the present model is the phase shift or lag in the particle entrainment rate, $\phi_{e q}$, with respect to the flow variables. This physical phenomenon was first introduced by Kennedy [8, 17] and can be described as a lag between the flow speed used to compute the entrainment rate, $\phi_{e q}(u(\hat{x}-\hat{\varphi} / \hat{k}, \hat{t}))$, and the actual water speed of that point, $u(\hat{x}, \hat{t})$. In accordance with Kennedy's [8] criterion, we set $-\pi / 2 \leq \hat{\varphi} \leq 0$ or $3 \pi / 2 \leq \hat{\varphi} \leq 2 \pi$ due to the periodic property of the wave. This lag, therefore, affects the term of the Taylor 

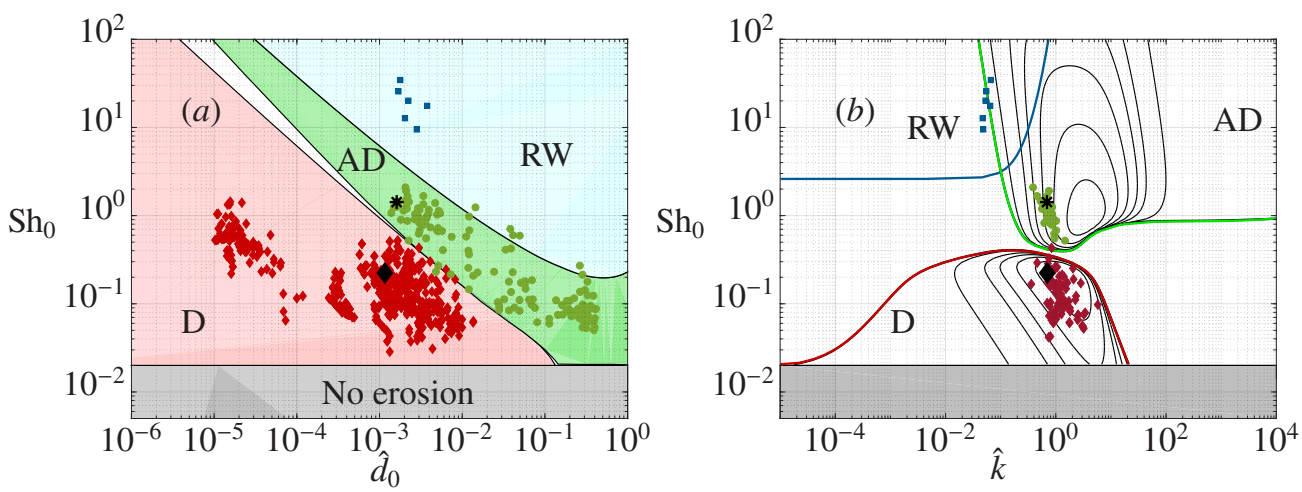

Figure 2. (a) Unstable region in the plane $\left\{\mathrm{Sh}_{0}, \hat{d}_{0}\right\}$ for values in the range of $\hat{k}$ observed in experiments. Red diamonds correspond to dunes (D) collected by Cheng [18] and Bradley \& Venditti [19], green circles correspond to antidunes (AD) collected by Recking et al. [20] and Carling \& Shvidchenko [21], and blue squares correspond to roll-wave experiments (RW) by Brock [22]. Also, the black diamond and asterisk respectively highlight the dune and antidune experiments by Naqshband et al. [23] and Simons [20] simulated in figure 3. (b) Contour lines of the constant growth rate in the $\left\{\mathrm{Sh}_{0}, \hat{k}\right\}$ plane for $\hat{d}_{0}=3 \times 10^{-3}$ and experiments with similar characteristic grain size. Thin contour lines: $\hat{\omega}_{i}=0.001,0.005,0.01,0.02,0.04,0.08$ for antidunes and one fourth of these values for dunes.

series expansion of $\phi_{e q}$ in (9)-(10), leading to the lagged speed $u^{\varphi} \equiv u(\hat{x}-\hat{\varphi} / \hat{k}, \hat{t})$. Note that $u^{\varphi}=U \exp [i(\hat{k} \hat{x}-\hat{\omega} \hat{t})] \exp (-i \hat{\varphi})$. Because of this, Cañada-Pereira \& Bohorquez [2] have proposed the phase-lag function to $\hat{\varphi}=\pi / 4[\tanh (10 \mathrm{Fr}-7)-1] \exp \left(-7.36 \hat{d}_{0}\right)$ which attenuates the magnitude of the phase lag as the grain roughness increases and cancels out in supercritical regime.

Evaluating the derivatives in time and space of the differential equations (7)-(10) for the wave term, $\exp [i(\hat{k} \hat{x}-\hat{\omega} \hat{t})]$, we obtain the eigenproblem $\mathbf{A} \cdot \mathbf{T}=\mathbf{0}$ :

$$
\begin{aligned}
& {\left[-i \hat{\omega}\left(\begin{array}{cccc}
1 & 0 & 0 & 0 \\
0 & 1 & 0 & 0 \\
0 & 0 & 1 & 0 \\
0 & 0 & 0 & \mathrm{Fr}^{2}
\end{array}\right)+i \hat{k}\left(\begin{array}{cccc}
0 & 0 & 0 & 0 \\
0 & \beta & 0 & \beta \\
0 & 0 & 1 & 1 \\
1 & 0 & 1 & \mathrm{Fr}^{2}
\end{array}\right)-\hat{k}^{2}\left(\begin{array}{cccc}
0 & 0 & 0 & 0 \\
0 & -\hat{D}_{u} & 0 & 0 \\
0 & 0 & 0 & 0 \\
0 & 0 & 0 & -\mathrm{Fr}^{2} \hat{v}
\end{array}\right)+\right.} \\
&\left.\left(\begin{array}{cccc}
0 & -\hat{\kappa}_{e} & 0 & \hat{\kappa}_{e} \phi_{e q}^{\prime} \exp (-i \hat{\varphi}) \\
0 & \hat{\kappa} & 0 & -\hat{\kappa} \phi_{e q}^{\prime} \exp (-i \hat{\varphi}) \\
0 & 0 & 0 & 0 \\
0 & 0 & -S_{0} & 2 S_{0}
\end{array}\right)\right] \cdot\left[\begin{array}{c}
\zeta \\
\Phi \\
\Gamma \\
U
\end{array}\right]=0,
\end{aligned}
$$

where the matrix $\mathbf{A}$ can be reduced to second order by means of the relation established between $\Gamma=f(U)$ and $\zeta=f(\Phi, U)$ described in previous works, see Bohorquez \& Ancey [10]. Note that the linear solutions to the problem share the term $\exp \left\{i\left[\hat{k} \hat{x}-\left(\hat{\omega}_{r}+i \hat{\omega}_{i}\right) \hat{t}\right]\right\}=$ $\exp \left[i\left(\hat{k} \hat{x}-\hat{\omega}_{r} \hat{t}\right)\right] \exp \left(\hat{\omega}_{i} \hat{t}\right)$ from which the amplification factor of the wave can be easily obtained, i.e. $\exp \left(\hat{\omega}_{i} \hat{t}\right)$. The base flow is unstable (stable) when the growth rate is positive, i.e. $\hat{\omega}_{i}>0$ (negative $\left.\hat{\omega}_{i}<0\right)$.

\section{Results}

There are only two independent parameters that characterise the stability of the base flow when solving for the eigenvalue problem (15), namely $\hat{d}_{0}$ and Fr. Alternatively, one can 

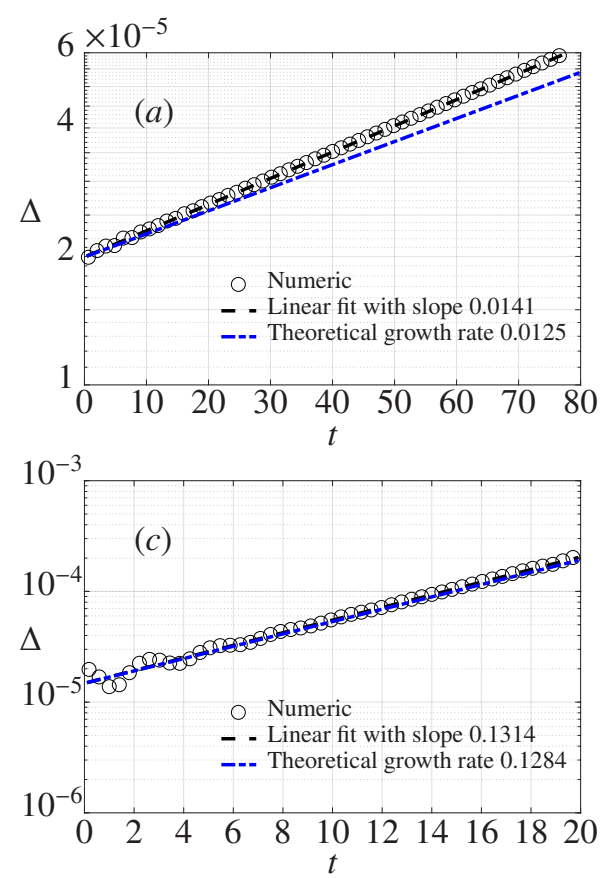
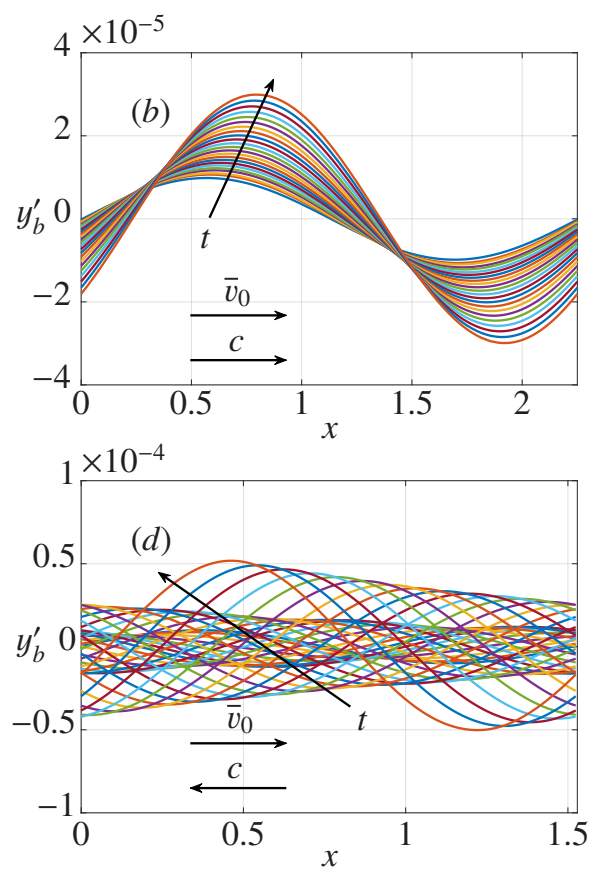

Figure 3. Non-linear numerical simulation of the growth of an infinitesimal perturbation of the bed with the initial amplitude of $2 \times 10^{-5} \mathrm{~m}$. (a) Maximum amplitude of the bed elevation, $\Delta=\max \left(y_{b}+\right.$ $\left.x S_{0}\right)-\min \left(y_{b}+x S_{0}\right)[\mathrm{m}]$, as a function of time $(t[\mathrm{~s}])$ for the dune experiment by Naqshband et al. [23]. The model parameters are $d=2.9 \times 10^{-4} \mathrm{~m}, h_{0}=0.25 \mathrm{~m}, \bar{v}_{0}=0.64 \mathrm{~m} \mathrm{~s}^{-1}, \Lambda=2 \pi h_{0} / \hat{k}=2.25 \mathrm{~m}$, $v_{t}=1, \mathrm{Sh}_{c r}=0.05, c_{d}=0.1, \mathrm{Sc}=0.5, \hat{\varphi}=3 \pi / 2$ and $\mathrm{Fr}=0.41$. (b) Profiles of the bed perturbation elevation from 0 to $80 \mathrm{~s}$. (c) Maximum amplitude of the bed elevation as a function of time for the experiment carried out by Simons. The model parameters are $d=2.7 \times 10^{-4} \mathrm{~m}, h_{0}=0.17 \mathrm{~m}, \bar{v}_{0}=1.5$ $\mathrm{m} \mathrm{s}^{-1}, \Lambda=1.53 \mathrm{~m}, v_{t}=1, \mathrm{Sh}_{c r}=0.05, c_{d}=0.1, \mathrm{Sc}=0.5, \hat{\varphi}=0$ and $\mathrm{Fr}=1.17$. (d) Profiles of the bed perturbation elevation from 0 to $15 \mathrm{~s}$.

select $\mathrm{Sh}_{0}$ instead of Fr using (12) with $u=1$. The neutral curves have been obtained by varying $\mathrm{Sh}_{0}$ and $\hat{d}_{0}$, and solving the dispersion relation (both real and imaginary part) for $\hat{\omega}$ and $\hat{k}$. The dispersion relation was obtained by setting the determinant of $\mathbf{A}$ to zero, i.e. $\mathbb{D}(\hat{k}, \hat{\omega}) \equiv|\mathbf{A}| \equiv 0$. It links the complex frequency, $\hat{\omega}$, to the real wavenumber, $\hat{k}$, and provides the bedform growth rate $\left(\hat{\omega}_{i}\right)$ and, consequently, the neutral curves $\left(\hat{\omega}_{i}=0\right)$ which determine the occurrence of roll wave, dune or antidune.

The solutions to the neutral curves lie in the parameter space $\left\{\mathrm{Fr}, \hat{k}, \hat{d}_{0}\right\}$ or $\left\{\mathrm{Sh}_{0}, \hat{k}, \hat{d}_{0}\right\}$ but, for the sake of simplicity, they have been projected onto the planes $\left\{\mathrm{Sh}_{0}, \hat{d}_{0}\right\}$ and $\left\{\mathrm{Sh}_{0}, \hat{k}\right\}$ in accordance with the bedforms diagrams by Carling \& Shvidchenko [21]. On the one hand, figure 2(a) shows the values of $\mathrm{Sh}_{0}$ for the range of wavenumbers $\hat{k}$ where the bulk of the experiments occur (i.e. $3 \times 10^{-1} \leq \hat{k} \leq 5$ for dunes and antidunes, and $4 \times 10^{-2} \leq \hat{k} \leq 7 \times 10^{-2}$ for roll waves). On the other hand, figure $2(b)$ draws the neutral curve and the growth rate in the $\{$ Sh, $\hat{k}\}$-plane at the specific value of $\hat{d}_{0}=3 \times 10^{-3}$, where the three types of instabilities co-exist. The distinction between both bedforms has been made regarding the sign of the wave speed $\left(\hat{c}=\hat{\omega}_{r} / \hat{k}\right)$, which is positive for downstream migrating dunes and negative for upstream migrating antidunes. The neutral curve for roll waves shares the positive wave speed but can be distinguished from the dune instability of the erodible bed due to the supercritical regime $(\mathrm{Fr}>1)$ of the base flow. 
In addition, the maximum and minimum values of $\mathrm{Sh}_{0}$ in the neutral curve of the dune and antidune is represented in figure $2(a)$ with solid lines as function of $\hat{d}_{0}$. Both curves nearly overlap for $\hat{d}_{0}>10^{-3}$. Also, the minimum value of $\mathrm{Sh}_{0}$ required for the development of roll wave is shown in solid line. Note that the curves $\operatorname{Sh}_{0}\left(\hat{d}_{0}\right)$ in figure $2(a)$ define four regions. The gray region corresponds to the absence of sediment motion (i.e. $\mathrm{Sh}_{0}<\mathrm{Sh}_{c r}$ ). Just above the threshold of sediment motion, dune or antidune occur depending on the value of $\mathrm{Sh}_{0}$, as depicted by the areas in red and green, respectively. Finally, the critical value for the free surface instability defines the blue region which lies above the antidune. In the three cases, a good agreement is observed with existing experiments (symbols).

At this point, it is worth mentioning that the wavenumber and Shields number observed in experiments with dunes and antidunes correspond to the most dangerous mode predicted by the linear stability theory. As a matter of fact, figure $2(b)$ shows that experimental points overlap with the regions of highest growth rate. It is fair to affirm that the stability theory correctly captures the wavelength selection mechanism observed in experiments.

In order to check the reliability of the proposed results, we benchmarked the linear stability results against non-linear simulations in the two following cases: first, we replicate the conditions in one of the antidune experiments carried out by Simons [20]; second, we mimic the experiment on dune by Naqshband et al. [23]. We followed the same procedure as described at length in Bohorquez \& Ancey [10]. We refer the reader to [10] for further details on the numerical scheme, boundary conditions and simulation set up. In both simulations we evaluated the dimensional growth rate, $\hat{\omega}_{i} h_{0} / \bar{v}_{0}\left[\mathrm{~s}^{-1}\right]$, by best fit of the bed elevation amplitude as a function of time, see figures $3(a)$ and $3(c)$. The numerical growth rate only differs from the linear theory by $11.35 \%$ for the dune experiment and $2.32 \%$ for the antidune. Therefore, the introduction of a phase lag in the model allows us to capture the bed instabilities in both subcritical and supercritical regimes with an accurate prediction of the linear growth rate. The agreement is higher for lower values of $\hat{\varphi}$ because the first-order term of the Taylor expansions of $\phi_{e q}^{\varphi}$ increases with $\hat{\varphi}$.

To conclude, figure $3(b)$ represents the evolution of the bed perturbation elevation in time for the dune, verifying the downstream migration direction as commonly observed in dunes. A different behaviour is obtained for antidunes, see figure $3(d)$, where the migration of the bedform occurs opposite to flow. These results have been corroborated by the linear stability theory, which shows a positive and negative value of $\hat{c}$, respectively.

\section{Conclusions}

In this paper, we have shown that the morphodynamic model by Bohorquez \& Ancey $[10,11]$ can be readily generalised to predict the formation of dunes at subcritical flow speed. To achieve this goal, one only needs to include a phase lag between the sediment entrainment rate and the depth-averaged flow velocity, as proposed by Cañada-Pereira \& Bohorquez [2]. We have formalised this great result by means of a simple linear stability theory. At the same time, the use of non-linear numerical simulations of the original model equations allowed us to verify the accuracy of the linear stability theory in realistic experimental conditions at the laboratory scale. Overall, for the first time, a morphodynamic model based on the shallowwater equations can predict the dune and antidune formation observed in one-dimensional flow experiments and natural phenomena.

The nondimensional groups controlling the erodible bed instability are the Shields (or Froude) numbers, Sh (Fr), the grain roughness relative to the flow depth, $\hat{d}_{0}$, and dimensionless wavenumber, $\hat{k}$. At subcritical speed, the particle diffusivity and the phase lag between erosion rate and flow velocity trigger destabilisation of the flat bed and the growth of dune 
while, for supercritical flow, the particle diffusivity provokes a wavelength selection of antidune in-phase with water wave. These arguments contrast with the common belief that the only model able to predict the dune-antidune instability is based on rotational or NavierStokes flow equation.

We conclude that erosion-deposition morphodynamic models based on the advectiondiffusion equation (Balmforth \& Vakil [24]; Ancey \& Heyman [12]; Bohorquez \& Ancey [10]) outperform the traditional Exner equation to study pattern formation. Taking into account the analogy of the morphodynamic model (1)-(4) and well-known non-equilibrium sediment transport equations, see Bohorquez \& Ancey [11], we point out that the inclusion of particle diffusivity and phase lag effects can be used to readily extend the capabilities of existing models [3]. Furthermore, depth-averaged equations offer the new possibility of simulating bedforms in large-scale geophysical flows as real rivers, which is particularly useful for practitioners of hydraulic engineering.

\section{References}

[1] A.J. Reynolds, J. Fluid Mech. 22, 113 (1965)

[2] P. Cañada-Pereira, P. Bohorquez, J. Fluid Mech. (2018), revised

[3] W. Wu, Computational River Dynamics (CRC Press, 2008)

[4] S. Niemann, J. Fredsøe, N. Jacobsen, J. Hydraul. Eng. 137, 5 (2010)

[5] O. van Duin, S. Hulscher, J. Ribberink, C. Dohmen-Janssen, J. Hydraul. Eng. 143, 04016084 (2017)

[6] M. Nabi, S. Giri, T. Iwasaki, I. Kimura, Y. Shimizu, Multi-scale modelling of river morphodynamics (2014), pp. 1253-1259

[7] A. Mendoza, J. Abad, E. Langendoen, D. Wang, P. Tassi, K. Abderrezzak, J. Hydraul. Eng. 143, 04016099 (2017)

[8] J.F. Kennedy, J. Fluid Mech. 16, 521 (1963)

[9] F. Charru, B. Andreotti, P. Claudin, Annu. Rev. Fluid Mech. 45, 469 (2013)

[10] P. Bohorquez, C. Ancey, Adv. Water Resour. 83, 36 (2015)

[11] P. Bohorquez, C. Ancey, Appl. Math. Model. 40, 7474 (2016)

[12] C. Ancey, J. Heyman, J. Fluid Mech. 744, 129 (2014)

[13] C. Ancey, P. Bohorquez, J. Heyman, J. Geophys. Res. Earth Surf. 120, 2529 (2015)

[14] E. Lajeunesse, L. Malverti, F. Charru, J. Geophys. Res. Earth Surf. 115, F04001 (2010)

[15] P.Y. Julien, Erosion and Sedimentation (Cambridge University Press, 2010)

[16] P.J. Schmid, D.S. Henningson, Stability and Transition in Shear Flows (Springer, 2001)

[17] J.F. Kennedy, Annu. Rev. Fluid Mech. 1, 147 (1969)

[18] N.S. Cheng, J. Hydraul. Eng. 142, 04016057 (2016)

[19] R.W. Bradley, J.G. Venditti, Earth-Sci. Rev. 165, 356 (2017)

[20] A. Recking, V. Bacchi, M. Naaim, P. Frey, J. Geophys. Res. Earth Surf. 114, F04025 (2009)

[21] P.A. Carling, A.B. Shvidchenko, Sedimentology 49, 1269 (2002)

[22] R.R. Brock, J. Hydraul. Div. 95, 1401 (1969)

[23] S. Naqshband, J. Ribberink, D. Hurther, S. Hulscher, J. Geophys. Res. Earth Surf. 119, 1043 (2014)

[24] N.J. Balmforth, A. Vakil, J. Fluid Mech. 695, 35 (2012) 